Gynäkol Geburtshilfliche Rundsch 1994;34:253

\title{
Autorenregister Vol. 34, 1994
}

Das Supplement 1 hat ein eigenes Autorenregister

Arbeitsgruppe HIV der Schwei-zerischen Gesellschaft für Gynäkologie und Geburts-

hilfe(SGGG) 186

Arzt,W. 54

Aspöck,H. 50

Bartussek,G. 131 Benz,D. 106 Bernet,R. 43 Bollmann,R. 145 Buddeberg,C. 43 Bustan, M.N. 134

Chaoui,R. 145 Coker,A.L. 134 Cooper, E.S. 134 Czembirek,H. 130

Dapunt,O. 168 David, M. 175 Daxenbichler, G. 168 DeGrandi,P. 7 Delucca,A. 191 Deutinger, J. 71 Dobec,M. 171 Drack,G. 91

Ebert,A. 175 Enzelsberger, H. 23

Fehr,P. 106

Fischer, M. 29 Frigg-Bützberger, A. 91 Frobenius, W. 58 Fuith,L. 131

Gaarenstroom, K.N. 133 Gaillard, M.-C. 7 Girardi,F. 162 Graf, A. 131

Grüninger, Th. 171 Güntert,B.J. 91

Haller,U. 1,79,137,157 Hammer, J. 102 Hanzal, E. 25 Hartl,J. 54 Hartmann, B.W. 189

Häusler,G. 25 Heling,K.S. 145 Helmer, H. 23 Hepp,H. 1,69,137 Heydarfadai, M. 162

Holböck,E. 55 Huber,J. 87 Huber,J.C. 189 Huch,A. 34 Husslein,P. 50,87

Janisch, H. 50 Joura, E. 25, 98

Kainz,Ch. 98 Kanhai, H.H.H. 133 Karas,H. 87 Keller, A. 171 Kiss,H. 87 Klein, M. 131

Köchli,O.R. 3,79,123,157 Kölbl,H. 25 Konecny,G 184 Koroschetz, F. 162 Krayenbühl, M. 34

Kucera,H. 131 Kulenkampff, K.J. 130 Küng,F. 157 Kurz,C. 23

Lahousen, M. 131 Landolt,U. 79 Lang,N. 58

Lehner,R. 87 Leodolter,S. 130,165 Lorenz, U. 106

Marth,C. 168 Matal,W. 29 Mayer, H. 131 Medl,M. 130,131, 165 Moroder, W. 191 Möse,J.R.

50 Müller,J. 106

Nagler-Reus, M. 109

Pädiatrische AIDS-Gruppe Schweiz (PAGS), Kommis-sion der Schweize-rischen Gesellschaft für Pädiatrie 186

Pakisch,B. 131

Patzen,M. 91

Peters-Engl, Ch. 130,165

Pickel,H. 162

Pieber,D. 32

Pollak,A. 50

Poschauko, J. 131

Ralph, G 27 Reinold,E. 1,137 Reinthaller, A. 98 Reus,W.A. 109 Rosen, A. 131 Ruschitzka, G. 102 
Sadoghi,H. 54 Schaller,A. 37 Schär,G 79 Schied,G. 27 Schneider, J. 152 Schoissengeier, A. 55 Schröck, A. 131

Seifert,B. 157

Selbmann, H.-K. 139

Sevin,B.U. 79

Slunsky,R. 17

Stiglbauer, M. 131

Stiskal,M. 130

Stummvoll,W. 55,102

Stürzbecher, M. 175

Subkommission Klinik (SKK) der Eidgenössischen Kommission für AIDS-Fragen(EKAF) 186 Szalay,S. 131

Tamussino, K. 27, 32 Tempfer, G 98 Tews, G 54

Trotsenburg, M. van 182 Tulusan,A.H. 58 Tulzer,G. 54

Vandenbussche, F.P.H.A. 133 Vander-Möse, A. 50 Vavra,N. 131 Vytiska,E. 87

Watson-Duff, E.M. 134 Weinberger, R. 54 Weissenbacher, E.R. 52 Wellenhofer, A. 131

Wenzl,R. 87 Widschwendter, M. 168 Wietlisbach, M. 152 Wimmer-Puchinger, B. 117 Winter, R. 50, 162 Wirrani,N. 131 Wolfram, G 131

Zimmermann, R. 34 Zivkovic, F. 27, 32

253

Sachregister Vol. 34,1994

Das Supplement 1 hat kein Sachregister

Antike geburtshilfliche Lehre 109 Arzt-Patientin-Beziehung 43

AsymptomatischeVarizelleninfektionen

171 ATP-Chemosensibilitätsassay 79

Bad des Neugeborenen 109 Beckenboden 25 Beckenbodengymnastik 32

Blasenentleerungsstörung nach Kolposus-

pension 106 Blutgerinnung, Ligatur 17 Britische Facharztausbildung 184 Brusttumoren 165

Chromosomenaberration 71 Cisplatin 79 Cockroft 157 Cyclosporin 34

Endometriumsablation 7 Endothelläsion 17

Europäische Niederlassungsrichtlinien 184 European College of Obstetrics and Gyne-cology 184 Facharztausbildung in Europa 182, 184 Farbdopplersonographie 165 Fetale Echokardiographie 145 - Herzbiometrie 145 FIGO-Einteilung 3

Gametentransfer 152 Geschichte der Medizin 37 Gynäkologisch-geburtshilflicheStatistiken 139 Hämostase 17 Harnblasenkapazität 23 Harninkontinenz 23,25, 32 Hebamme 91

Hebammenschulen 91 HIV 186 Hysteroskopie 7

IgM-Bestimmungen 171 Informationsverarbeitung 139 Inkontinenzfragebogen 25

Inkontinenzoperation 29 Interferon 168 Intratubaler Raum 87

Jelliffe 157

Kolporrhaphie 27 Kongenitale Herzfehler 145 Konnatale Infektionen 171 Konsensus

Ovarialkarzinom 123 Kreatinin-Clearance 157 Kunstgeschichte 109

Laparoskopie 152 Lebertransplantation 34 Loop-Excision 162

Maligne Trophoblasterkrankungen 3 Medizingeschichte 175 Messerkonisation 162 Micholitsch

37 Mythologie 109

Nachwuchsbedarf 91 Nd-Yag-Laser 7

Neoplasie, zervikale intraepitheliale 98 Neurourodynamik 27 NIH-Klassifikation 3 
Obstruktive Miktionsstörung 106 Ovarialkarzinom 157 Oxybutynintherapie 23

Patientinnenzufriedenheit 117 Pränatale Diagnostik 145 Psychologische Qualitätsstandards 117

Radiochirurgie 98 Regionale Anästhesie 152 Resektoskop 7 Rezidivinkontinenz 29 Royal

College of Obstetrics and Gynaecology 184

Schwangerenbetreuung 117 Schwangerschaft 34,186 Schwangerschaftserleben 117

Schwangerschaftsinfektions-Screening 171 Standard-Heparin, niedrigdosierte Prophylaxe 17 Sterilität 87 Stressinkontinenz 27

Taxol 79

Transurethrale Blasenhalsresektion 106

Triple-Test 71

Trisomie 2171

Tuboskopie 87

Ultraschall 71, 165

Umfassendes Qualitätsmanagement 139 Urethraunterfütterung 29 Uterinagefásse 102

Vaginale Farbdopplersonographie 102 Vaginalkonus 32 Vitamin-A-Säure 168

Weibel 37 Wertheim 37 WHO-Scoringsystem 3

Zervixkarzinom 102 Zidovudin 186

254 